\title{
Throughput Optimization for Multipath Unicast Routing Under Probabilistic Jamming
}

\author{
Patrick Tague, Sidharth Nabar, James A. Ritcey, David Slater, and Radha Poovendran \\ Network Security Lab (NSL), Department of Electrical Engineering \\ University of Washington, Seattle, WA, USA. \\ Email: \{tague, snabar, jar7, dmslater, rp3\}@u.washington.edu
}

\begin{abstract}
We present a framework for throughput optimization for multipath unicast routing in wireless networks in the presence of probabilistic jamming. The framework introduces a statistical characterization into the maximum network flow problem to compensate for the reduction in network flow due to the loss of jammed packets. We map the problem of throughput optimization under probabilistic jamming to that of optimal investment portfolio selection, treating the network throughput as the return on financial investments and using a common portfolio selection framework from financial statistics. Based on the portfolio selection framework, we present approaches to maximize expected throughput and to minimize throughput variance. We include both a detailed example and a simulation study to illustrate the application of the throughput optimization framework.
\end{abstract}

\section{INTRODUCTION}

One of the most effective denial-of-service (DoS) attacks [1] on a wireless communication network is jamming. Recent work has demonstrated that in addition to jamming attacks targeting the physical layer [2], an intelligent jammer can formulate an attack by targeting certain link layer and MAC implementations [3]-[5], link layer error detection and correction protocols [6], and routing protocols [7] by taking advantage of protocol details and cross-layer information leakage. The availability of higher-layer information allows a jammer to perform an efficient attack, even under the use of physical layer anti-jamming techniques such as spreadspectrum communication. Hence, even a resource constrained jammer can perform such attacks. We note that jammers can also operate effectively on underwater acoustic networks [8].

Since the primary goal in network communication is to deliver source data to nodes throughout the network, we are interested in the problem of maximizing network throughput in the presence of jamming. When a jammer is present in the network, jamming attacks which target different layers

This work was supported in part by the following grants: ARO PECASE, W911NF-05-1-0491; ONR, N000-07-1-0600; ARL CTA, DAAD19-01-20011; and ARO MURI, W911NF-07-1-0287.

This document was prepared through collaborative participation in the Communications and Networks Consortium sponsored by the US Army Research Laboratory under the Collaborative Technology Alliance Program, DAAD19-01-2-0011. The US Government is authorized to reproduce and distribute reprints for Government purposes notwithstanding any copyright notation thereon. The views and conclusions contained in this document are those of the author and should not be interpreted as representing the official policies, either expressed or implied, of the Army Research Laboratory or the US Government. of the network protocol stack have a direct impact on the performance of the routing protocol and the ability to deliver data throughout the network. Existing techniques for routing under jamming provide methods for reaction to jamming by classifying each receiving node as either jammed or not jammed. For example, the authors of [5] provide a technique to estimate and route around the region of the network being jammed, and the authors of [9] propose an approach to detect jamming and perform coordinated channel-hopping.

In this work, we propose a method to build on these approaches for reactive routing by incorporating a statistical characterization of the jamming attack into the routing protocol. Based on our model, using statistical information about the probabilistic jammer, the source node can introduce bias into the routing decisions, leading to a preference to route data through regions of the network that have been jammed less frequently in the recent past. The use of the available statistics thus lead to proactive improvement in network throughput.

Our contributions to the problem of optimizing network throughput for multipath unicast routing in the presence of probabilistic jamming are summarized as follows.

- We incorporate the effect of probabilistic jamming into the network flow problem thus compensating for the random loss of packets due to jamming.

- Based on the network flow formulation, we present a framework for throughput optimization for multipath routing under probabilistic jamming, making use of portfolio theory from financial statistics [10], [11].

- We provide examples and simulation to illustrate the optimal achievable throughput using different statistical utility functions based on the expected throughput and the throughput variance to capture the network dynamics under probabilistic jamming.

The remainder of this paper is organized as follows. Section II states assumptions about the network model and the jamming attack. Section III formulates a new framework for routing optimization under jamming. Section IV provides a detailed example to illustrate the use of the optimization framework. Section V evaluates the performance of the proposed approach via simulation. In Section VI, we summarize our results. 


\section{NETWORK MOdEL}

The wireless network consists of a set $\mathcal{N}$ of nodes with pairwise communication represented by a directed graph $G=$ $(\mathcal{N}, \mathcal{E})$ such that a pair $(i, j)$ is in $\mathcal{E}$ if and only if node $j$ can receive packets directly from node $i$. We assume that all nodeto-node communication is unicast, i.e. each packet transmitted by a node $i \in \mathcal{N}$ is intended for a unique $j \in \mathcal{N}$ with $(i, j) \in$ $\mathcal{E}$. For each $(i, j) \in \mathcal{E}$, we denote the maximum achievable data rate, or capacity, of the link in the absence of jamming as $c_{i j}$. At a given instant in time, a source node $s \in \mathcal{N}$ generates data to be routed to a destination node $d \in \mathcal{N}$. The decisions used to route data from a given source $s$ to the destination $d$ at a given time instant depend on the statistical model used to characterize the active jamming attack.

In this work, we assume that the network nodes have no information about the specific jamming attack being performed by the adversary. We instead model the effect of jamming by defining the probability $x_{j} \in[0,1]$ that each receiving node $j$ will correctly receive a transmitted packet. Due to the network's uncertainty in the adversary's actions, each probability $x_{j}$ can itself be characterized as a random variable using statistics from past network communication. We denote the expected value and variance of each random variable $x_{j}$ as $\mu_{j}$ and $\sigma_{j}^{2}$, respectively.

We assume that the source node $s$ is able to collect information about the end-to-end routing topology, including link capacities and statistics (e.g. mean, variance, confidence intervals) on each of the random probabilities $x_{j}$ for relevant nodes $j$. We thus formulate the optimal throughput routing problem with respect to the flow allocation by the source $s$ using this available information.

\section{Network Throughput Optimization FRAMEWORK}

In this section, we present a framework for network throughput optimization under active jamming attacks. We first develop the constraints imposed on the routing solutions and then propose a variety of utility functions to determine optimality of solutions.

\section{A. Optimization Constraints}

In order to define a set of constraints for the throughput optimization problem, we must consider both the link capacity constraints in the wireless network and the effect of the adversarial jamming attack. Let $f_{i j}$ denote the total flow rate transmitted onto the link $(i, j) \in \mathcal{E}$ by node $i \in \mathcal{N}$. Given the fixed network topology and link capacities $c_{i j}$, any network flow solution must satisfy the capacity constraints

$$
0 \leq f_{i j} \leq c_{i j} \text { for all }(i, j) \in \mathcal{E} .
$$

However, the total flow rate received by node $j \in \mathcal{N}$ from the link $(i, j)$ may not be equal to $f_{i j}$ due to the jamming attack. In particular, the received rate by $j$ is reduced by the random fraction $x_{j}$. The total flow intended for each receiving node $j \in \mathcal{N}$ is randomly reduced due to the jamming adversary, implying that flow is not perfectly conserved throughout the network. To incorporate the random loss of data into the network flow model, we formulate the problem with respect to the flow rate sent along each routing path from $s$ to $d$ instead of the rate over each link $(i, j)$.

Let $\mathcal{P}=\left\{p_{1}, \ldots, p_{L}\right\}$ denote the collection of paths used to route packets from $s$ to $d$. Each path $p_{\ell} \in \mathcal{P}$ is given by a subset $p_{\ell} \subseteq \mathcal{E}$ of directed links in the network and is assumed to be loop-free. We let $\phi_{\ell}$ denote the intended flow rate along each path $p_{\ell} \in \mathcal{P}$ as indicated by the source node $s$. The total data rate of the source $s$ is thus given by the summation of $\phi_{\ell}$ over $\ell=1, \ldots, L$. Since the total flow $f_{i j}$ on each edge $(i, j)$ is equal to the summation of path flows $\phi_{\ell}$ for each path $p_{\ell}$ traversing the edge, the capacity constraint in (1) can be written as

$$
0 \leq \sum_{\ell:(i, j) \in p_{\ell}} \phi_{\ell} \leq c_{i j} \text { for all }(i, j) \in \mathcal{E} .
$$

Letting $\phi$ denote the $L \times 1$ column vector of rates $\phi_{\ell}$, we see that this constraint is linear in the variable $\phi$.

In traversing the link $(i, j) \in \mathcal{E}$, the data rate $r$ transmitted by node $i$ is reduced to a received rate of $x_{j} r$ at node $j$. Hence, the achieved throughput along the path $p_{\ell}$ from $s$ to $d$ is equal to $\phi_{\ell} \prod_{(i, j) \in p_{\ell}} x_{j}$. For each $\ell=1, \ldots, L$, we thus define the fraction of intended flow along $p_{\ell}$ that successfully reaches $d$ as the random fraction $y_{\ell}$ given by

$$
y_{\ell}=\prod_{(i, j) \in p_{\ell}} x_{j} .
$$

We denote the expected value and variance of each variable $y_{\ell}$ as $\gamma_{\ell}$ and $\omega_{\ell}^{2}$, respectively. Due to the fact that a pair of paths in $\mathcal{P}$ may visit a common receiving node $j \in \mathcal{N}$, the path random variables $y_{\ell}$ may be correlated even if the node random variables $x_{j}$ are mutually independent. Hence, in addition to the variance $\omega_{\ell}^{2}$ of each random variable $y_{\ell}$, we define the $L \times$ $L$ covariance matrix $\Omega$ to characterize the correlation among paths in $\mathcal{P}$.

We next provide a generalization of the link capacity constraint in (2) to incorporate both the link capacities $c$ and the jamming statistics of the random variables $x$. This generalization is motivated by noting that jamming effectively reduces the data flow rate along each path $p_{\ell}$ as the distance from the source $s$ increases. Hence, if the data flow rate at the source $s$ is chosen according to (2), the actual data flow rate $f_{i j}$ on each edge $(i, j) \in \mathcal{E}$ may not be tightly bound by the capacity $c_{i j}$. Instead, we are interested in a formulation that proactively adjusts the sending rate along each path $p_{\ell}$ to compensate for the data losses due to jamming at downstream receivers and to allocate bandwidth to flows in regard to the statistical behavior of the residual flow rate. If a downstream bottleneck link exists, instead of limiting the sending data rate to match the capacity of the bottleneck link, the data rate can be increased so the residual flow over the bottleneck is approximately equal to its capacity, thereby compensating for the data rate reduction due to jamming.

We thus generalize (2) by replacing the flow $f_{i j}$ on each link $(i, j)$ by a statistic $\mathcal{G}_{i j}$ which characterizes the behavior of the 
data flow on the link. The function $\mathcal{G}_{i j}$ takes the corresponding entries of the flow vector $\phi$ and the jamming random variables $x$ as parameters. The generalized capacity constraint is thus given by

$$
0 \leq \mathcal{G}_{i j}(\phi, x) \leq c_{i j} \text { for all }(i, j) \in \mathcal{E} .
$$

By inspection, we see that the original link capacity constraint in (2) is included as a special case of this formulation.

In this paper, we define the statistic $\mathcal{G}_{i j}$ as the expected residual flow on the edge $(i, j)$ after packet loss due to jamming, capturing the average-case effect of the active jamming attack. The average-case capacity constraint is obtained by defining the expected residual flow $\mathcal{G}_{i j}(\phi, x)$ as

$$
\mathcal{G}_{i j}(\phi, x)=\sum_{\ell:(i, j) \in p_{\ell}} \phi_{\ell} E\left[\prod_{\substack{k: p_{\ell} \text { visits } \\ k \text { before } j}} x_{k}\right],
$$

where $E[\cdot]$ denotes expected value with respect to $x$. Due to the use of averaging in this constraint, nodes in the network must be allowed to buffer excess data, thereby introducing delay into the data flow. We do not address the effect of such delay, leaving it as future work.

\section{B. Utility Functions Based on Portfolio Selection Theory}

Given the constraint statistic $\mathcal{G}_{i j}$ discussed in Section III-A, the remaining part of the optimization formulation is the choice of a utility function to be maximized. A statistical utility function $\mathcal{U}(\phi)$ to be maximized can be any function of the assigned path flow rates $\phi$ and random parameters $x$. Since the role of routing is to deliver data to destination nodes, we consider utility functions related to the total data rate, or throughput, delivered to $d \in \mathcal{N}$ as a function of the flow assignment $\phi$ and the jamming parameters $x$. The throughput $T(y)$ as a function of the path random variables $y$ is equal to the vector inner product $\phi^{T} y$. Letting $\gamma$ denote the $L \times 1$ vector of means $\gamma_{\ell}$ of the random variables $y_{\ell}$, we use portfolio selection theory to define utility functions using the expected throughput $\phi^{T} \gamma$ and the throughput variance $\phi^{T} \Omega \phi$.

1) Maximum Expected Throughput: As a non-deterministic extension of the maximum flow problem, we first define the utility function $\mathcal{U}_{T}(\phi)$ as the expected throughput $\mathcal{U}_{T}(\phi)=$ $\phi^{T} \gamma$ achieved by the allocation of flow rates $\phi$. This maximum expected throughput, or maxT, formulation yields the following optimization problem.

\begin{tabular}{cl}
\multicolumn{2}{c}{ Maximum Expected Throughput $(\max T)$} \\
\hline $\max _{\phi}$ & $\mathcal{U}_{T}(\phi)=\phi^{T} \gamma$ \\
s.t. & $0 \leq \mathcal{G}_{i j}(\phi, x) \leq c_{i j}$ for all $(i, j) \in \mathcal{E}$, \\
& $\phi \geq 0$. \\
\hline
\end{tabular}

The maximum expected throughput formulation estimates the maximum achievable throughput for a given routing topology. However, the objective function $\mathcal{U}_{T}(\phi)$ does not capture the degree of variation in the throughput, as indicated by the variance $\phi^{T} \Omega \phi$. If this variance is high, the uncertainty in the estimated maximum achievable throughput is high. In order to include the throughput variance in the problem formulation, we use portfolio selection theory [10], [11] from financial statistics, in which variance is interpreted as financial risk.

2) Minimum Throughput Variance with Bounded Mean: A common formulation to evaluate the risk of a financial allocation to correlated assets is to use Markowitz's portfolio selection theory [10], [11]. The goal is to minimize the variance subject to a constraint on the expected value, leading to the analogous minimum throughput variance, or $\min \mathbf{V}$, formulation. In this throughput optimization formulation, the set $\mathcal{P}$ of routing paths is treated as a portfolio of assets, and the flow allocation vector $\phi$ is treated as the financial allocation to the assets in the portfolio. The achieved throughput is interpreted as the investment return. The utility function $\mathcal{U}_{V}(\phi)$ to be maximized is thus given by the negative variance

$$
\mathcal{U}_{V}(\phi)=-\phi^{T} \Omega \phi .
$$

To prevent the trivial solution $\phi=0$ with minimum variance 0 , an additional lower bound constraint is imposed on the expected throughput, given by

$$
\phi^{T} \gamma \geq T_{\min }>0 .
$$

The resulting optimal throughput routing formulation is thus given by the solution to the following optimization problem.

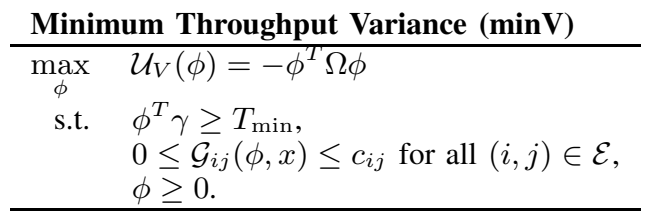

As an alternate formulation, the fixed lower bound $T_{\min }$ can be replaced by a variable expected throughput that is simultaneously optimized with the throughput variance as follows.

3) Optimal Trade-off of Throughput Mean and Variance: Simultaneous maximization of the expected throughput and minimization of the throughput variance can be achieved by the following alternate formulation of the Markowitz portfolio selection problem. Instead of forcing the expected throughput to be lower-bounded by $T_{\min }$, we trade the utility obtained by maximizing the expected throughput with that of minimizing the throughput variance using a trade-off parameter $\eta$. The utility function $\mathcal{U}_{\eta}(\phi)$ which realizes this mean-variance tradeoff is given by

$$
\mathcal{U}_{\eta}(\phi)=\eta \phi^{T} \gamma-\phi^{T} \Omega \phi .
$$

By adjusting the trade-off parameter $\eta$, the weight on each of the expected throughput and throughput variance can be changed. This mean-variance throughput trade-off, or MVtrade, formulation for a given parameter $\eta$ is thus given by the following optimization problem.

\begin{tabular}{cl} 
Mean-Variance Throughput Trade-off (MVtrade) \\
\hline $\max _{\phi}$ & $\mathcal{U}_{\eta}(\phi)=\eta \phi^{T} \gamma-\phi^{T} \Omega \phi$ \\
s.t. & $0 \leq \mathcal{G}_{i j}(\phi, x) \leq c_{i j}$ for all $(i, j) \in \mathcal{E}$, \\
& $\phi \geq 0$. \\
\hline
\end{tabular}




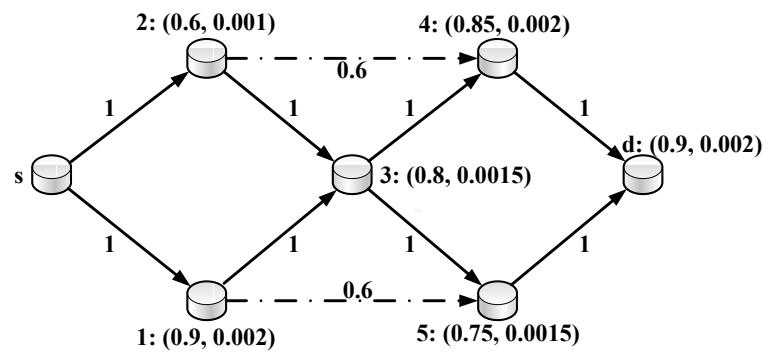

Fig. 1. The example network is illustrated. The source node is labeled with identifier $s$, and each receiving node $j \in \mathcal{N}$ is labeled $j:\left(\mu_{j}, \sigma_{j}^{2}\right)$, where $\mu_{j}$ and $\sigma_{j}^{2}$ are the mean and variance of the beta random variable $x_{j}$ characterizing the jamming statistics of node $j$.

For the constraint statistic $\mathcal{G}_{i j}$ given by (5) for each link $(i, j) \in \mathcal{E}$, each of the maximum expected throughput, minimum throughput variance, and mean-variance throughput trade-off problems can be solved in polynomial time using interior-point methods for linear and quadratic programming [11]. In general, the solutions can be found in polynomial time as long as $\mathcal{G}_{i j}$ is linear in the optimization variable $\phi$.

\section{EXAMPLE}

In this section, we provide an example to illustrate the application of the throughput optimization framework presented in Section III.

The example network is given in Fig. 1, with the label on each link $(i, j)$ indicating the link capacity $c_{i j}$. The source node is labeled with identifier $s$, and each receiving node is labeled with an identifier $j \in \mathcal{N}$ and the mean $\mu_{j}$ and variance $\sigma_{j}^{2}$ of the jamming parameter $x_{j}$. In this example, we assume that each jamming parameter $x_{j}$ is distributed according to an independent beta random variable [12] with parameters $\left(\alpha_{j}, \beta_{j}\right)$ related to $\mu_{j}$ and $\sigma_{j}^{2}$ as

$$
\begin{aligned}
\mu_{j} & =\frac{\alpha_{j}}{\alpha_{j}+\beta_{j}} \\
\sigma_{j}^{2} & =\frac{\alpha_{j} \beta_{j}}{\left(\alpha_{j}+\beta_{j}+1\right)\left(\alpha_{j}+\beta_{j}\right)^{2}} .
\end{aligned}
$$

For example, node 1 with $\mu_{1}=0.9$ and $\sigma_{1}^{2}=0.002$ has corresponding parameters $\alpha_{1}=39.6$ and $\beta_{1}=4.4$.

In this example, we consider the set $\mathcal{P}$ of $L=4$ paths. For each path $p_{\ell} \in \mathcal{P}$, we associate the random variable $y_{\ell}$ given by (3) as the product of corresponding beta random variables $x_{j}$. Assuming the variables $x_{j}$ for $j \in \mathcal{N}$ are independent, we compute the corresponding means $\gamma_{\ell}$ and variances $\omega_{\ell}^{2}$ of $y_{\ell}$. The paths in $\mathcal{P}$ and the corresponding $\gamma_{\ell}$ and $\omega_{\ell}^{2}$ values are as follows.

\begin{tabular}{c|c|c|c}
\hline Path & Nodes Included & $\gamma_{\ell}$ & $\omega_{\ell}^{2}$ \\
\hline$p_{1}$ & $s, 2,4, d$ & 0.4590 & 0.0017 \\
$p_{2}$ & $s, 1,5, d$ & 0.6075 & 0.0028 \\
$p_{3}$ & $s, 2,3,5, d$ & 0.3240 & 0.0011 \\
$p_{4}$ & $s, 1,3,4, d$ & 0.5508 & 0.0031 \\
\hline
\end{tabular}

Each off-diagonal covariance in $\Omega$ can be computed from means $\mu_{j}$ and variances $\sigma_{j}^{2}$ based on the common nodes in the two corresponding paths. For example, paths $p_{1}$ and $p_{3}$ have nodes 2 and $d$ in common and nodes 3,4 , and 5 not in common, so the covariance $\omega_{13}=E\left[y_{1} y_{3}\right]-E\left[y_{1}\right] E\left[y_{3}\right]$ is given by $\omega_{13}=\mu_{3} \mu_{4} \mu_{5}\left(\sigma_{2}^{2}+\mu_{2}^{2}\right)\left(\sigma_{d}^{2}+\mu_{d}^{2}\right)-\gamma_{1} \gamma_{3}$.

By inspection of the network topology in Fig. 1, we see that in the absence of jamming, a total network flow of $\phi_{1}+\phi_{2}+$ $\phi_{3}+\phi_{4}=2$ units can be achieved by choosing $\phi_{1}+\phi_{3}=$ $\phi_{2}+\phi_{4}=1$ with $\phi_{1} \leq 0.6$ and $\phi_{2} \leq 0.6$. This baseline flow allocation is thus compared to that resulting from each optimization formulation using the average-case function $\mathcal{G}$ given by (5). We compare the resulting flow allocations for the maximum expected throughput (maxT) formulation, the minimum throughput variance (minV) formulation, and the mean-variance throughput trade-off (MVtrade) formulation.

For each of the three optimization problems, we determine the optimal flow allocation vector $\phi$ and compute the expected throughput $E[T(x)]$ and the throughput variance $V[T(x)]$. The following results illustrate the maxT results, three minV results for $T_{\min }=0.6,0.8,1.0$, and three $\mathbf{M V t r a d e}$ results for $\eta=10^{-4}, 10^{-3}, 10^{-2}$.

\begin{tabular}{c|c|c}
\hline Formulation & $E[T(x)]$ & $V[T(x)]$ \\
\hline $\max$ & 1.048 & $5.82 \times 10^{-3}$ \\
$\min \mathbf{}, T_{\min }=0.6$ & 0.600 & $1.85 \times 10^{-3}$ \\
$\min \mathbf{}, T_{\min }=0.8$ & 0.800 & $3.29 \times 10^{-3}$ \\
$\min \mathbf{}, T_{\min }=1.0$ & 1.000 & $5.25 \times 10^{-3}$ \\
MVtrade, $\eta=10^{-4}$ & 0.019 & $1.94 \times 10^{-6}$ \\
MVtrade, $\eta=10^{-3}$ & 0.194 & $1.94 \times 10^{-4}$ \\
MVtrade, $\eta=10^{-2}$ & 1.048 & $5.82 \times 10^{-3}$ \\
\hline
\end{tabular}

From the given results, we make the following observations. The maxT formulation provides an upper bound on the achievable expected throughput under jamming. The minV formulation yields expected throughput equal to the lower bound $T_{\min }$ but with a significant reduction in throughput variance compared to the maxT formulation. As the bound $T_{\min }$ increases toward the optimal maxT result, the variance increases correspondingly. The MVtrade formulation balances the trade-off between throughput mean and variance as a function of the bias parameter $\eta$. For small values of $\eta$, the MVtrade formulation primarily minimizes the variance, leading to low mean throughput. As $\eta$ increases, the utility is weighted more heavily on the mean, so the result approaches that of the maxT formulation.

\section{Performance Evaluation}

In this section, we perform a simulation study to evaluate the performance of the optimal throughput routing formulations proposed in Section III. In this simulation study, we fix the network topology with $|\mathcal{N}|=25$ nodes as given in Fig. 2. In the figure, the network topology is illustrated by including a dotted line to represent each edge $(i, j) \in \mathcal{E}$, and all edges have the same capacity of 0.1 units. The set $\mathcal{P}$ consists of $L=10$ paths chosen as a subset of the possible paths joining the source node $s$ and destination node $d$.

For each of the optimization problems presented in Section III, we simulate 500 trials, each corresponding to a different set of jamming statistics at each node $j \in \mathcal{N}$. As in Section IV, we model the jamming parameter $x_{j}$ for each 


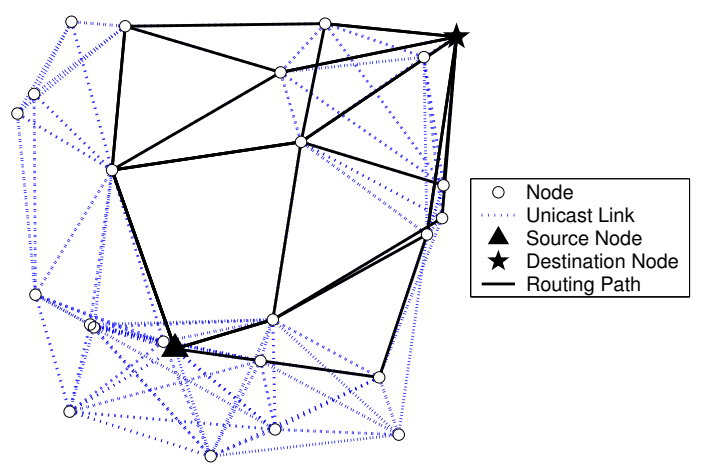

Fig. 2. The simulation study uses the given network of $|\mathcal{N}|=25$ nodes with $L=10$ paths. The simulation results reflect variation in the jamming statistics at each node to reflect the achievable throughput profile of the fixed network.

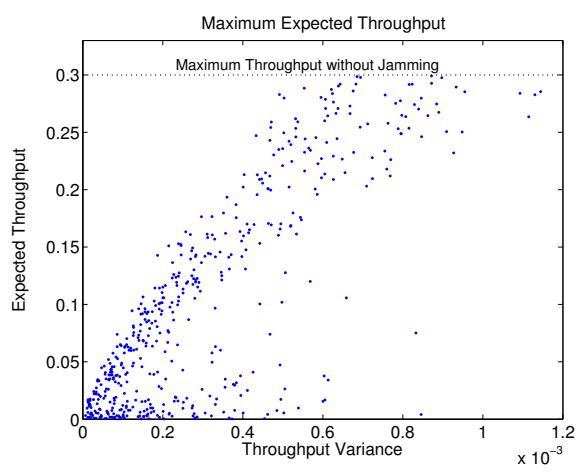

Fig. 3. The maxT formulation is evaluated by comparing the expected throughput and throughput variance for the network in Fig. 2. Each data point represents a different set of jamming statistics $x_{j}$ for $j \in \mathcal{N}$.

node $j$ as a beta random variable with mean $\mu_{j}$ and variance $\sigma_{j}^{2}$. The jamming parameter $y_{\ell}$ for each path $p_{\ell} \in \mathcal{P}$ is modeled as the corresponding random variable as described in Section IV. The capacity constraints in our simulation use the statistic $\mathcal{G}_{i j}$ given by (5). The performance of the maxT formulation is illustrated in Fig. 3, comparing the achievable throughput means and variances. We see that the variance tends to increase with the mean, clearly illustrating the tradeoff between throughput mean and variance. We next evaluated the performance of the $\mathbf{m i n} \mathbf{V}$ formulation using the additional lower bound $T_{\min }$ on the expected throughput. The result of this problem formulation is that the lower bound is achieved with equality and the throughput variance is driven nearly to 0 , similar to the results in Section IV. Due to page limitations, supporting figures are not included.

We evaluate the performance of the MVtrade formulation for several trade-off parameters $\eta$ to vary the balance between maximum mean throughput and minimum throughput variance. From the figure, we see that the optimal solution for each set of jamming statistics falls on a line with slope inversely proportional to $\eta$. This result illustrates the trade-off between the expected throughput and the throughput variance and shows that the trade-offs are easily quantifiable for this problem formulation.

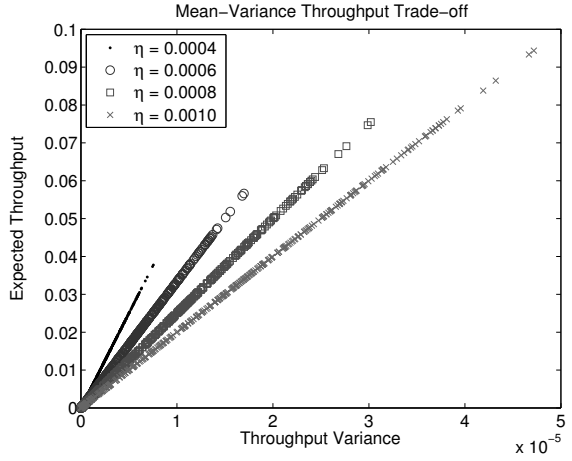

Fig. 4. The MVtrade formulation is evaluated by comparing the expected throughput and throughput variance for the network in Fig. 2. Each curve reflects a different value of $\eta$ as indicated.

\section{CONCLUSION}

In this work, we proposed a framework for throughput optimization in multipath unicast routing in the presence of an adaptive jammer. We incorporated a statistical characterization of the jamming attack into the maximum network flow problem to compensate for expected losses. This work allows for proactive routing under jamming by incorporating the statistics of the past jamming events into route selection. We made use of portfolio selection theory from financial statistics, treating the network throughput as the return on a financial investment portfolio and derived utility functions based on the throughput mean and variance. Our future work will extend the formulation to a more general network routing model.

\section{REFERENCES}

[1] R. Anderson, Security Engineering: A Guide to Building Dependable Distributed Systems. Wiley, 2001.

[2] R. A. Poisel, Modern Communication Jamming Principles and Techniques. Artech House, 2004.

[3] J. Bellardo and S. Savage, "802.11 denial-of-service attacks: Real vulnerabilities and practical solutions," in Proc. USENIX Security Symposium, Washington, DC, Aug. 2003, pp. 15-28.

[4] D. J. Thuente and M. Acharya, "Intelligent jamming in wireless networks with applications to $802.11 \mathrm{~b}$ and other networks," in Proc. 25th IEEE Communications Society Military Communications Conference (MILCOM'06), Washington, DC, Oct. 2006, pp. 1-7.

[5] A. D. Wood and J. A. Stankovic, "Denial of service in sensor networks," IEEE Computer, vol. 35, no. 10, pp. 54-62, Oct. 2002.

[6] G. Lin and G. Noubir, "On link layer denial of service in data wireless LANs," Wireless Communications and Mobile Computing, vol. 5, no. 3 , pp. 273-284, May 2005.

[7] P. Tague, D. Slater, G. Noubir, and R. Poovendran, "Modeling optimal jamming attacks on network traffic flows," in Proc. 6th International Symposium on Modeling and Optimization in Mobile, Ad Hoc, and Wireless Networks (WiOpt'08), Berlin, Germany, Apr. 2008.

[8] E. M. Sozer, M. Stojanovic, and J. G. Proakis, "Underwater acoustic networks," IEEE Journal of Oceanic Engineering, vol. 25, no. 1, pp. 72-83, Jan. 2000.

[9] W. Xu, K. Ma, W. Trappe, and Y. Zhang, "Jamming sensor networks: Attack and defense strategies," IEEE Network, vol. 20, no. 3, pp. 41-47, May/Jun. 2006.

[10] H. Markowitz, "Portfolio selection," The Journal of Finance, vol. 7, no. 1, pp. 77-92, Mar. 1952.

[11] S. Boyd and L. Vandenberghe, Convex Optimization. Cambridge, 2004.

[12] D. J. C. MacKay, Information Theory, Inference, and Learning Algorithms. Cambridge: Cambridge University Press, 2003. 